\title{
Atmospheric Error Correction of the Laser Beam Ranging
}

\author{
J. Saydi, ${ }^{1}$ A. Lotfalian, ${ }^{2}$ M. Abedi, ${ }^{2}$ J. Khalilzadeh, ${ }^{1}$ and H. Saghafifar ${ }^{3}$ \\ ${ }^{1}$ Physics Department, Imam Hosein University, P.O. Box 16575-347, Tehran, Iran \\ ${ }^{2}$ Malek-Ashtar University of Technology, Isfahan, Iran \\ ${ }^{3}$ Center of Optics and Laser Researches, Malek-Ashtar University of Technology, Isfahan, Iran \\ Correspondence should be addressed to J. Khalilzadeh; jkhalil@ihu.ac.ir
}

Received 24 April 2014; Revised 28 July 2014; Accepted 12 August 2014; Published 7 September 2014

Academic Editor: Hesham El-Askary

Copyright (C) 2014 J. Saydi et al. This is an open access article distributed under the Creative Commons Attribution License, which permits unrestricted use, distribution, and reproduction in any medium, provided the original work is properly cited.

\begin{abstract}
Atmospheric models based on surface measurements of pressure, temperature, and relative humidity have been used to increase the laser ranging accuracy by ray tracing. Atmospheric refraction can cause significant errors in laser ranging systems. Through the present research, the atmospheric effects on the laser beam were investigated by using the principles of laser ranging. Atmospheric correction was calculated for $0.532,1.3$, and 10.6 micron wavelengths through the weather conditions of Tehran, Isfahan, and Bushehr in Iran since March 2012 to March 2013. Through the present research the atmospheric correction was computed for meteorological data in base of monthly mean. Of course, the meteorological data were received from meteorological stations in Tehran, Isfahan, and Bushehr. Atmospheric correction was calculated for 11, 100, and 200 kilometers laser beam propagations under $30^{\circ}, 60^{\circ}$, and $90^{\circ}$ rising angles for each propagation. The results of the study showed that in the same months and beam emission angles, the atmospheric correction was most accurate for 10.6 micron wavelength. The laser ranging error was decreased by increasing the laser emission angle. The atmospheric correction with two Marini-Murray and Mendes-Pavlis models for $0.532 \mathrm{~nm}$ was compared.
\end{abstract}

\section{Introduction}

Through the laser range finding systems, the distance was calculated by computation of the round-trip time of emitted beam and reflected from the target. From this point of view, this class of systems had two basic parts: the pulse generator and emitter including its optical system and attached equipment and detector and received pulses analyzer including receiving telescope and beam time of flight measuring unit [1]. The laser range finder system at large distances was developed and used in the USA between 1961 and 1962 for first time for satellites ranging specially. The applied satellite was BE-B (BEACONEXPLORER-B). The first successful received signal was achieved in 1965 . The atmosphere through the most recent models has been assumed to be symmetric and spherical to simplify the calculations of range finding and delay caused by atmosphere. In these models, horizontal gradients in atmosphere index of reflection would be ignored because it led to error less than a centimeter in angels less than 10 degrees [2]. Due to the atmosphere and turbulence, beam refraction induced error through the laser range finding systems. Atmospheric refraction index increased the mean optical path. The group velocity error ranged from 2.5 meters at zenith to almost 13 meters at elevation of 10 degrees. Intense atmospheric turbulences caused a random error in the optical path length which probably would be a few centimeters at 10 degrees. Many models have been developed to correct laser range measurements because of mean atmospheric index of refraction. The atmospheric measuring data resulting from range finding sites were used through the mentioned models and their accuracy received a few centimeters at 20 degrees. In order to increase the accuracy of these measurements, it was shown that the accuracy was limited by the atmospheric propagation and turbulence effects and also by the system hardware. For single-frequency satellite laser ranging systems, Marini and Murray proposed a model containing the precision requirements at that time. Therefore, all known developments of the improved atmospheric correction formulae have been based on the MariniMurray scheme [3-5]. Mendes and Pavlis developed a new 


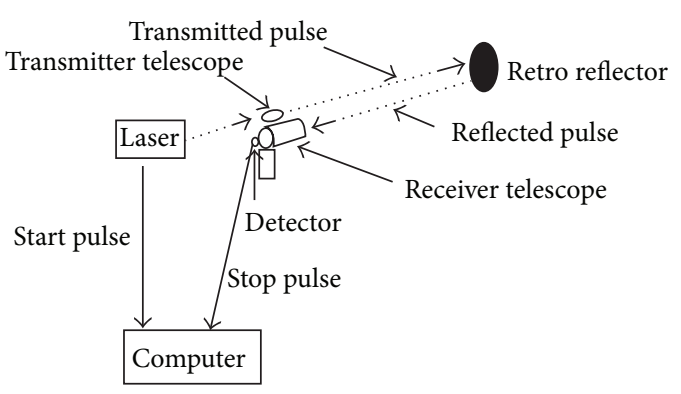

FIGURE 1: Laser tracking system scheme.

modified model based on the Marini-Murray [6]. For gradients with horizontal refraction and also for refractions with spherical symmetry, many accurate models could be used to correct errors. Of course, the mentioned models required many meteorological data. The models based on the surface measurements depended on near earth surface topography and other atmospheric effects. For its high accuracy, the laser range finding was one of the best range measuring methods $[7,8]$. The mentioned technique was used to air born platform ranging at short ranges and to space born platform ranging at long ranges. Refractive index difference between layers of atmosphere was one of the error factors in the accurate range finding. The atmospheric correction method was applied in order to minimize errors and compute accurate distance between ground site and target. Through the present method, to achieve a real value of the distance between ground site and target, the error value has been calculated and encountered in the final measurement due to atmospheric effects (AC). Through the present work, the atmospheric effects on laser propagation were investigated based on the Marini-Murray model. Group refractive index error was calculated by using the Mendes and Pavlis modified model for comparison. Due to atmosphere, the occurred error on accurate measuring has been studied considering weather conditions of laser emitter site. The atmospheric correction for weather conditions in one year of Tehran, Isfahan, and Bushehr was calculated. Weather conditions, that is, mean pressure, mean temperature, mean precipitation, and mean relative humidity were $664(\mathrm{mmHg}), 18^{\circ} \mathrm{C}$, $225 \mathrm{~mm}$, and $41 \%$, for Tehran, $630(\mathrm{mmHg}), 16^{\circ} \mathrm{C}, 160 \mathrm{~mm}$, and $52 \%$ for Isfahan, and $760(\mathrm{mmHg}), 25^{\circ} \mathrm{C}, 280 \mathrm{~mm}$, and $71 \%$ for Bushehr, respectively. The atmospheric correction was calculated for laser beams at several wavelengths and for 11,100 , and $200 \mathrm{~km}$ propagation path lengths. Calculations were done for three laser beam emission angles, that is, 30, 60 , and 90 degrees. Because of this, the real meteorological data of the above mentioned states was used.

\section{Laser Range Finding Fundamentals}

In laser range finding of distance between a ground site and a target, a very short laser pulse is guided to a telescope for sending to target. Sent pulses back-scatter from the target surface to receiver telescope. This telescope is equipped to a beam recording detector which determines pulse time of

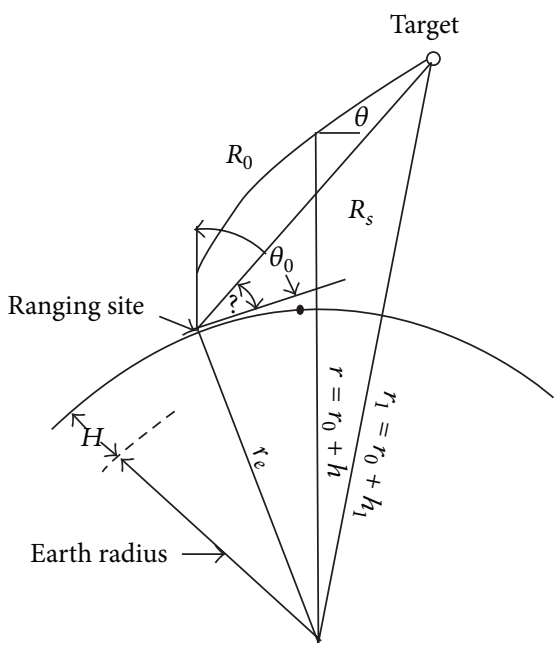

FIGURE 2: Curved and straight path of beam.

fight. Figure 1 shows a schematic diagram of this system. The measured time multiplied by speed of light gives two time of distance between laser emitter site and the target $[9,10]$. Because of large distance between ground site and target, the pick power of pulse must be large enough to reach reflecting surface and then back scatter to detector. One of the important parameters in accurate measuring is the pulse width of the sent pulse. Thus a very short pulse with width of about a few tens picoseconds is selected. According to $\tau \times c / 2,0.45 \mathrm{~cm}$ measuring accuracy will be achieved with a 30 picosecond pulse width [1].

\section{Atmospheric Correction Theory}

The laser beam which is the same as the other electromagnetic radiations was affected by its propagating medium. When the light transmits from a medium to another one, its speed was varied. Instead of the direct propagation of light path, the mentioned speed variation caused refraction or concaving of it. Rate of the concave merely was affected by the concentration difference of two mediums and frequency (or wavelength) of light. The earth atmosphere was not a homogeneous medium. It also included gases and other fine particles, making evaluating medium variation effect complicated. This nonhomogeneity of medium caused refraction of beam in propagation path. This path at long distances became concave. Figure 2 shows the concaved path of beam $\left(R_{0}\right)$ and straight path $\left(R_{s}\right)$ that will travel in vacuum. The traveled optical path by laser beam can be evaluated from the following equation [11]:

$$
R_{0}=\int_{r_{0}}^{r_{1}} \frac{n_{g}}{\sin \theta} d r,
$$

where, $n_{g}$ is the group refractive index and $\theta$ is the angle that could be evaluated by Snell's law for a layered spherical medium using (2):

$$
n r \cos \theta=n_{0} r_{0} \cos \theta_{0},
$$




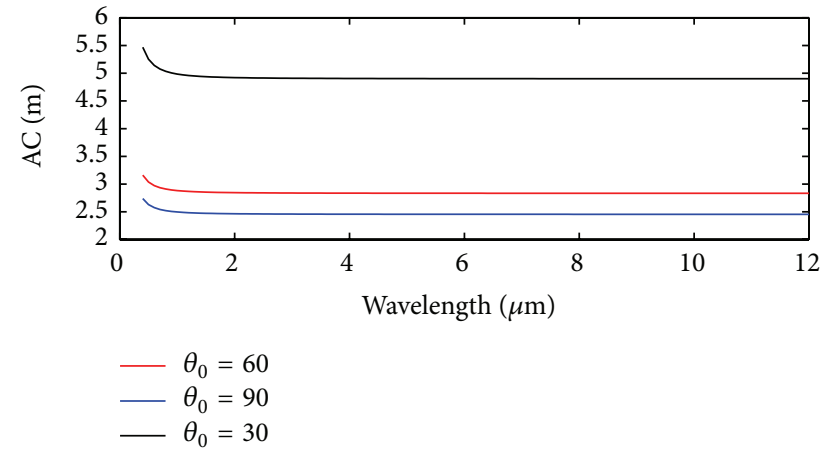

FIGURE 3: Wavelength dependence of AC in Isfahan weather conditions.

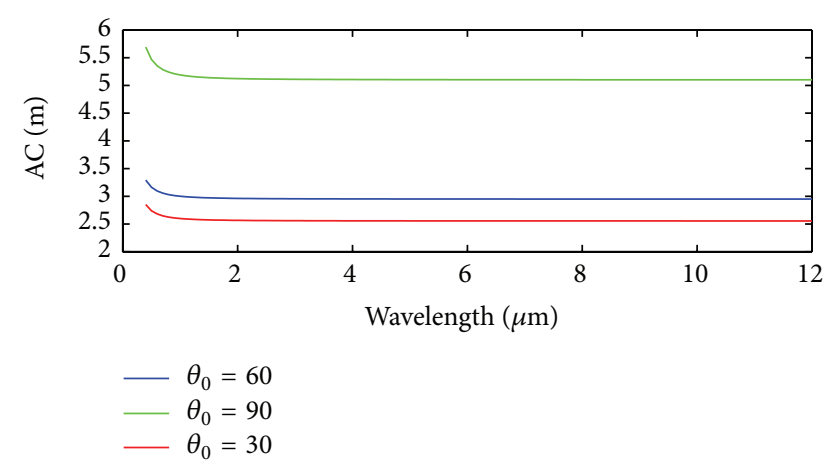

FIgure 4: Wavelength dependence of AC in Tehran weather conditions.

where, $n_{0}$ is refractive index and $\theta_{0}$ is the rising angle of beam at measuring site according to Figure 2. Group refractive index $\left(n_{g}\right)$ can be evaluated from the following equation [12]:

$$
n_{g}=1+10^{-6} N_{g}
$$

where, $N_{g}$ is the group refractive parameter. Calculating group refractive index, atmospheric correction (AC) can be achieved from (4):

$$
\begin{aligned}
\mathrm{AC} & =R_{0}-R_{s} \\
& =10^{-6} \int_{r_{0}}^{r_{1}} \frac{N_{g}}{\sin \theta} d r+\left[\int_{r_{0}}^{r_{1}} \frac{d r}{\sin \theta}-R_{s}\right] .
\end{aligned}
$$

First term in (4) is the group velocity error. Due to the variation of laser, the mentioned error is pulse group velocity which is because of atmospheric transmittance. Second term is the difference between direct beam path $\left(R_{s}\right)$ and beam traveled optical path $\left(R_{0}\right)$ as indicated in Figure 2. Because of the second term, the error value is about $3-4 \mathrm{~cm}$ for 10 degrees rising angle. For grater angles, it is negligible compared to first term error [11-13].

The group refractive parameter $N_{g}$ can be evaluated by Marini and Murray relation [14]:

$$
N_{g}=\left(\frac{273.15}{1013.25} \frac{P}{T} n_{g s}\right)-11.27\left(\frac{e}{T}\right),
$$

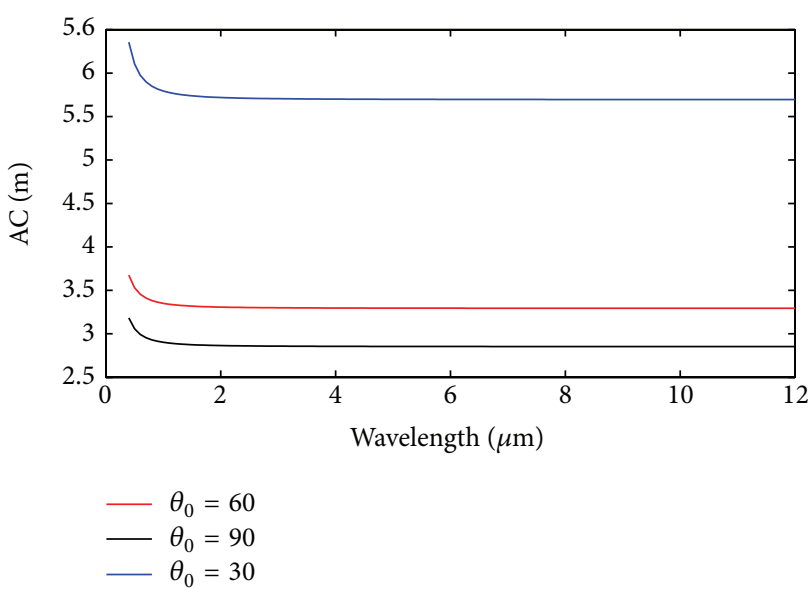

FIgURE 5: Wavelength dependence of AC in Bushehr weather conditions.

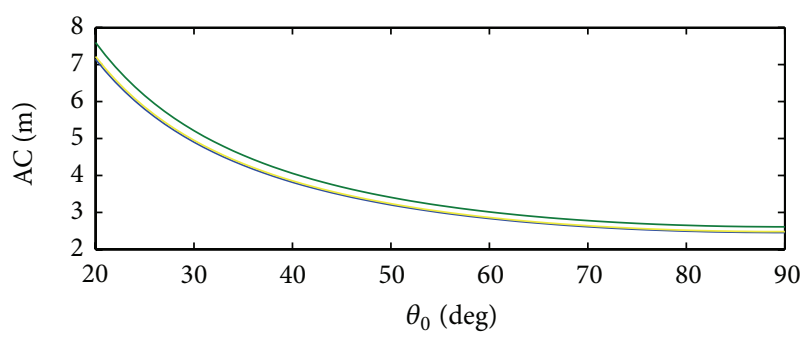

$\begin{aligned} \text { Wavelength } & =10.6 \mu \mathrm{m} \\ \text { Wavelength } & =1.3 \mu \mathrm{m} \\ \text { Wavelength } & =.532 \mu \mathrm{m}\end{aligned}$

FIgure 6: Rise angle $\left(\theta_{0}\right)$ dependence of AC in Isfahan weather conditions (two curves are overlapped and are not distinguishable).

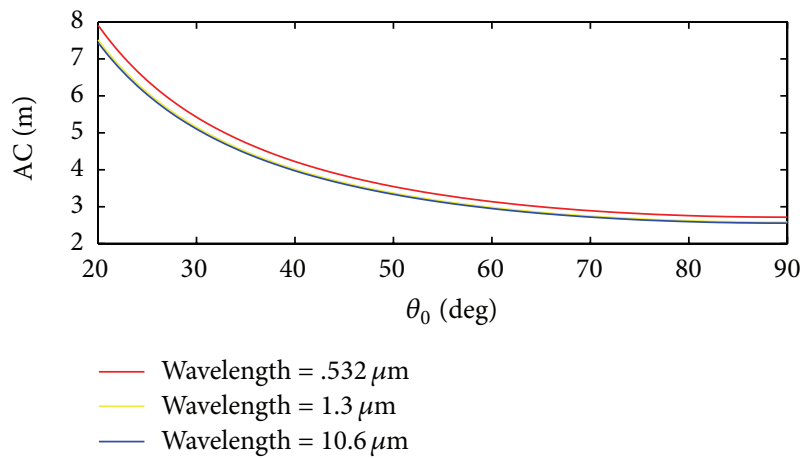

Figure 7: Rise angle $\left(\theta_{0}\right)$ dependence of AC in Tehran weather conditions (two curves are overlapped and are not distinguishable).

where $P$ is the overall pressure in hectopascal, $T$ is the temperature in Kelvin, $e$ is the partial water vapor pressure, and $n_{g s}$ is the group refraction index at standard conditions; that is, $T=273.15 \mathrm{~K}$ and $1 \mathrm{~atm}$. pressure. $n_{g s}$ can be evaluated 


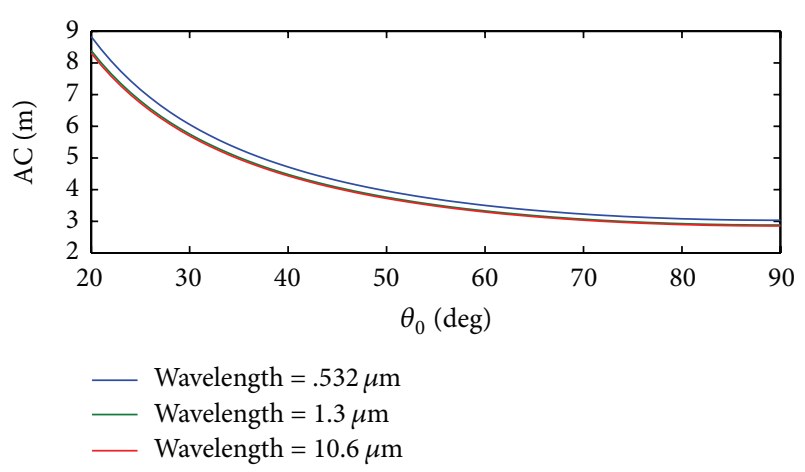

FIgURE 8: Rise angle $\left(\theta_{0}\right)$ dependence of AC in Bushehr weather conditions (two curves are overlapped and are not distinguishable).

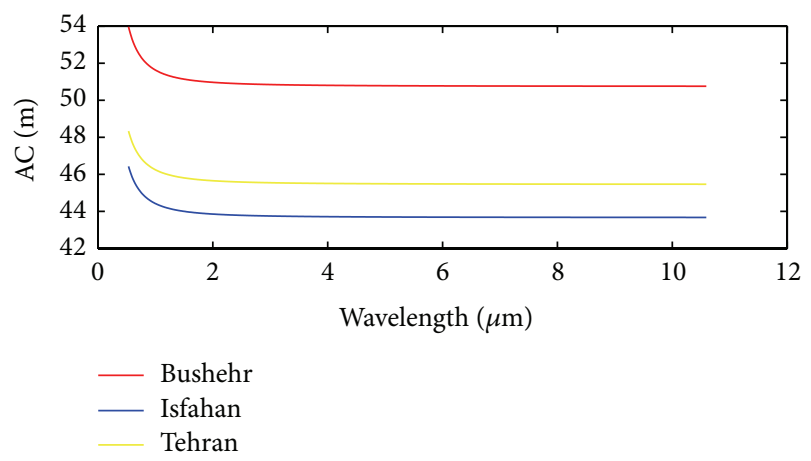

FIGURE 9: AC versus wavelength for $100 \mathrm{~km}$ altitude and laser beam emission angle of 30 degrees.

using (6) in which $\lambda$ is laser range finder wavelength in micrometer [15]. Consider the following:

$$
\begin{gathered}
n_{g s}=287.6155+\frac{4.8866}{\lambda^{2}}+\frac{0.068}{\lambda^{4}}, \\
e=0.01 \exp ^{\alpha}, \\
\alpha=1.2378847 \times 10^{-5} T^{2}-1.9121316 \times 10^{-2} T \\
+33.93711047-6.34316453 \times 10^{3} T^{-1} .
\end{gathered}
$$

\section{Results and Discussion}

AC value of Tehran, Isfahan, and Bushehr states was computed by using (1)-(8) and real meteorological monthly average data, that is, temperature, pressure, and relative humidity, during one year. AC value for the laser beams was calculated at $0.532,1.3$, and 10.6 microns and for 11,100 , and $200 \mathrm{~km}$ propagation path lengths. Calculations were done for three laser beam emission angles, that is, 30,60, and 90 degrees. Results are presented in Figures 3, 4, 5, 6, 7, 8, 9, $10,11,12,13$, and 14 . One can see from (4) and results show that the $\mathrm{AC}$ value obviously depends on the laser wavelength, emission angle, and propagation path length. As it can be seen through Figures 3-14, the atmospheric correction for $10.6 \mu \mathrm{m}$ at same emission angles and same propagation path length was the least one with respect to the other wavelengths. This

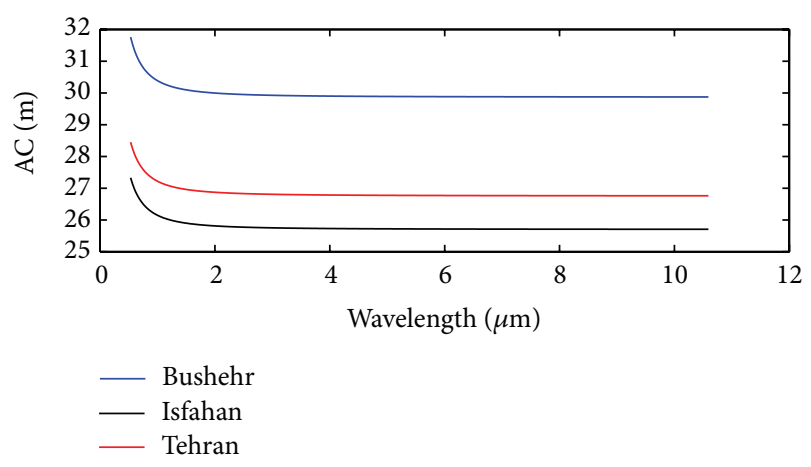

FIGURE 10: AC versus wavelength for $100 \mathrm{~km}$ altitude and laser beam emission angle of 60 degrees.

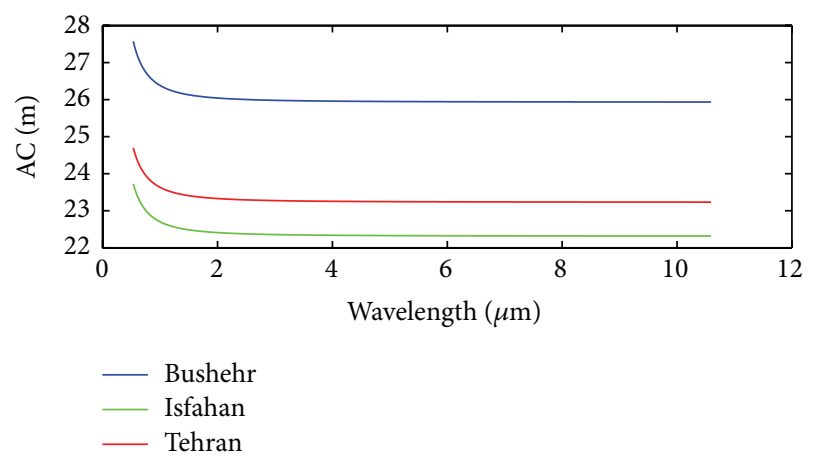

FIGURE 11: AC versus wavelength for $100 \mathrm{~km}$ altitude and laser beam emission angle of 90 degrees.

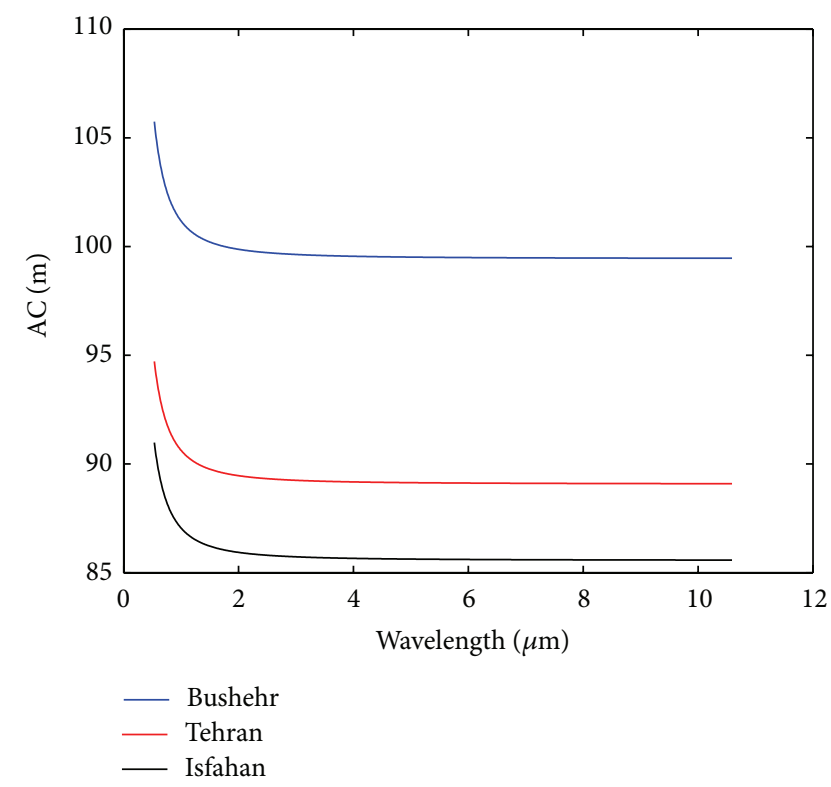

FIgURE 12: AC versus wavelength for $200 \mathrm{~km}$ altitude and laser beam emission angle of 30 degrees.

means that the $10.6 \mu \mathrm{m}$ needs less atmospheric correction. In the same manner $1.3 \mu \mathrm{m}$ needs less atmospheric correction with respect to $0.532 \mu \mathrm{m}$ wavelength. Figures $3-5$ and Figures 9-14 are atmospheric corrections versus wavelength curves. These curves are plotted for 11,100 , and $200 \mathrm{~km}$ propagation path lengths under three laser beam emission angles of 30 , 


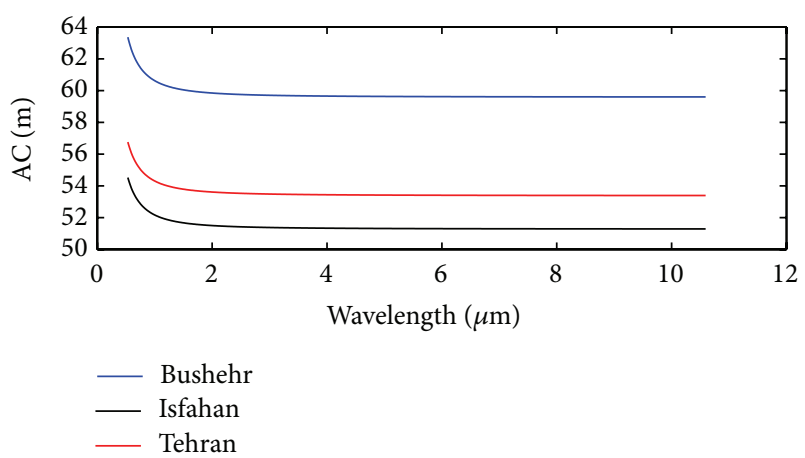

FIGURE 13: AC versus wavelength for $200 \mathrm{~km}$ altitude and laser beam emission angle of 60 degrees.

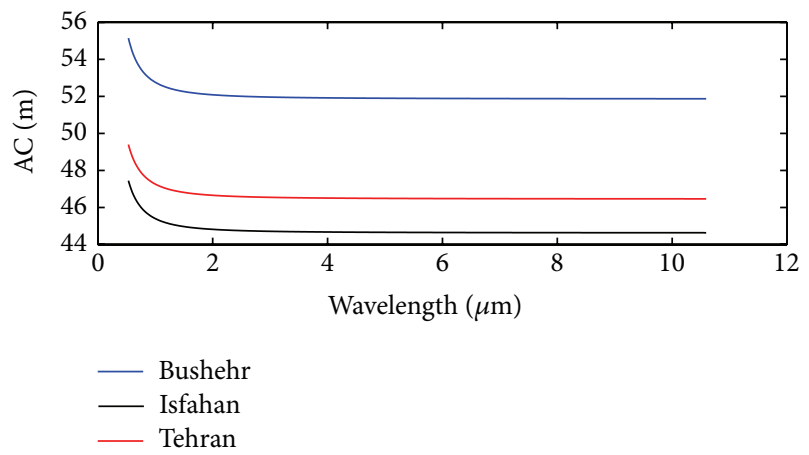

FIgURE 14: AC versus wavelength for $200 \mathrm{~km}$ altitude and laser beam emission angle of 90 degrees.

60 , and 90 degrees, respectively. These curves were figured for three numbered states (Tehran, Isfahan, and Bushehr) by utilizing metrological organization data.

According to (4) and (2), the AC value depends on the rising angle $\theta_{0}$, too. This dependency can be seen qualitatively in Figures 6-8. Results show that for a given propagation path length, increasing the rising angle decreases the atmospheric correction (see Figures 6-14). Atmospheric effect on laser beams is also investigated. Figures $6-8$, depict atmospheric correction versus rising angle $\theta_{0}$ diagrams for $0.532,1.3$, and $10.6 \mu \mathrm{m}$ wavelengths at $11 \mathrm{~km}$ propagation path length for three states. The group refraction parameter versus wavelength in Isfahan, Tehran, and Bushehr states weather conditions were calculated and are depicted in Figure 15. Mendes and Pavlis modified model has been used for AC correction of $0.532 \mu \mathrm{m}$ range finding and its results were compared with results of Marini-Murray model, which is depicted in Table 1. AC values in Table 1 show a distinguishable difference between two models and better results for Mendes and Pavlis model.

\section{Conclusion}

Through the present paper, the laser range finding system was investigated. Also errors due to the laser plus width and atmospheric effects at metrological conditions of laser beam emitter site were studied. Atmospheric correction for

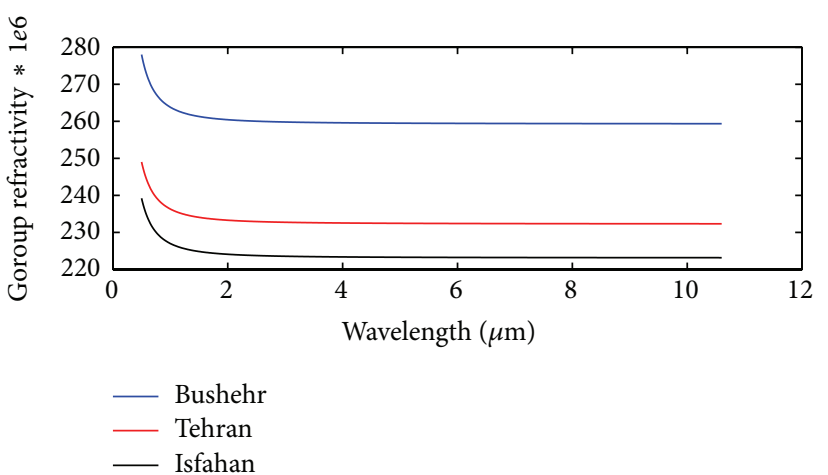

FIGURE 15: Group refraction parameter versus wavelength in Isfahan, Tehran, and Bushehr states weather conditions.

TABLE 1: AC comparison for two different applied models at $532 \mathrm{~nm}$ wavelength and $0^{\circ}$ zenith angle.

\begin{tabular}{|c|c|c|c|}
\hline City & Used model & Atmospheric path $(\mathrm{km})$ & $\mathrm{AC}(\mathrm{m})$ \\
\hline \multirow{6}{*}{ Tehran } & \multirow{3}{*}{ Mendes-Pavlis } & 11 & 2.2166 \\
\hline & & 100 & 2.3242 \\
\hline & & 200 & 2.3932 \\
\hline & \multirow{3}{*}{ Marini-Murray } & 11 & 2.5942 \\
\hline & & 100 & 23.5834 \\
\hline & & 200 & 47.1667 \\
\hline \multirow{6}{*}{ Esfahan } & \multirow{3}{*}{ Mendes-Pavlis } & 11 & 2.1756 \\
\hline & & 100 & 2.2323 \\
\hline & & 200 & 2.2985 \\
\hline & \multirow{3}{*}{ Marini-Murray } & 11 & 2.4898 \\
\hline & & 100 & 22.6349 \\
\hline & & 200 & 45.2698 \\
\hline \multirow{6}{*}{ Bushehr } & \multirow{3}{*}{ Mendes-Pavlis } & 11 & 2.6355 \\
\hline & & 100 & 2.7031 \\
\hline & & 200 & 2.7832 \\
\hline & \multirow{3}{*}{ Marini-Murray } & 11 & 2.7562 \\
\hline & & 100 & 25.0566 \\
\hline & & 200 & 50.1131 \\
\hline
\end{tabular}

monthly average metrological data of Tehran, Isfahan, and Bushehr states were calculated during a year. The atmospheric correction was calculated for $0.532,1.3$, and $10.6 \mu \mathrm{m}$ laser wavelengths and 11,100 , and $200 \mathrm{~km}$ propagation path lengths under three emission angles. The atmospheric correction values at same emission angles and propagation path lengths were increased from $10.6 \mu \mathrm{m}$ to $0.532 \mu \mathrm{m}$; therefore, it was concluded that the atmosphere is approximately a window for first wavelength. For given states, the differences between AC values showed that it depended on the atmospheric conditions, that is, temperature and pressure of measuring site. According to the accessed results, the minimum group refraction parameter was for Isfahan and maximum for Bushehr. It was concluded that the weather conditions of Bushehr, that is, high pressure, high temperature, and high humidity, corresponded to the high group parameter and consequently high group refractive index. This high group 
refractive index needs more atmospheric correction for the laser beams. The overall conclusion is that the various weather conditions cause error in the laser range finding and must be calculated and compensated.

\section{Conflict of Interests}

The authors declare that there is no conflict of interests regarding the publication of this paper.

\section{References}

[1] L. Combrinck, Satellite Laser Ranging, Sciences of Geodesy-I, Springer, Berlin, Germany, 2010.

[2] Z. Altamimi, P. Sillard, and C. Boucher, "ITRF2000: a new release of the International Terrestrial Reference Frame for earth science applications," Journal of Geophysical Research B: Solid Earth, vol. 107, no. 10, pp. 2-19, 2002.

[3] D. D. Wijaya and F. K. Brunner, "Atmospheric range correction for two-frequency SLR measurements," Journal of Geodesy, vol. 85, no. 9, pp. 623-635, 2011.

[4] J. W. Marini, "Correction of satellite tracking data for an arbitrary tropospheric profile," Radio Science, vol. 7, pp. 223-231, 1972.

[5] J. W. Marini and C. W. Murray, "Correction of laser range tracking data for atmospheric refraction at elevations above 10 degrees," NASA Technical Memorandum NASA-TM-X-70555, 1973.

[6] V. B. Mendes and E. C. Pavlis, "High-accuracy zenith delay prediction at optical wavelengths," Geophysical Research Letters, vol. 31, no. 14, 2004.

[7] H. J. Rim and B. E. Schutz, Geoscience Laser Altimeter System (GLAS), Algorithm Theoretical Basis Document, Version 2.2, The University of Texas at Austin, Austin, Tex, USA, 2002.

[8] G. C. Hulley and E. C. Pavlis, "A ray-tracing technique for improving Satellite Laser Ranging atmospheric delay corrections, including the effects of horizontal refractivity gradients," Journal of Geophysical Research B: Solid Earth, vol. 112, article B6, 2007.

[9] J. J. Degnan, "Asynchronous laser transponders for precise interplanetary ranging and time transfer," Journal of Geodynamics, vol. 34, no. 3-4, pp. 551-594, 2002.

[10] W. Colin and J. Julian, Handbook of Laser Technology, IOP Press, Bristol, UK, 2004.

[11] A. B. Mark, Development of the portable satellite laser ranging system [M.S. Thesis], Curtin University of Technology, 2003.

[12] S. Svanberg, Atomic and Molecular Spectroscopy: Basic Aspects and Practical Applications, Springer, New York, NY, USA, 4th edition, 2003.

[13] J. J. Degnan, "Satellite laser ranging current status and future prospects," IEEE Transactions on Geoscience and Remote Sensing, vol. 23, no. 4, pp. 398-413, 1985.

[14] J. B. Abshire and C. S. Gardner, "Chester, Atmospheric refractivity correction in satellite laser ranging," IEEE Transactions on Geoscience and Remote Sensing, vol. 23, no. 4, pp. 414-425, 1985.

[15] V. B. Mendes and E. C. Pavlis, "Atmospheric refraction at optical wavelengths: problems and solutions," in Proceedings of the 13th International Laser Ranging Workshop, 2002. 

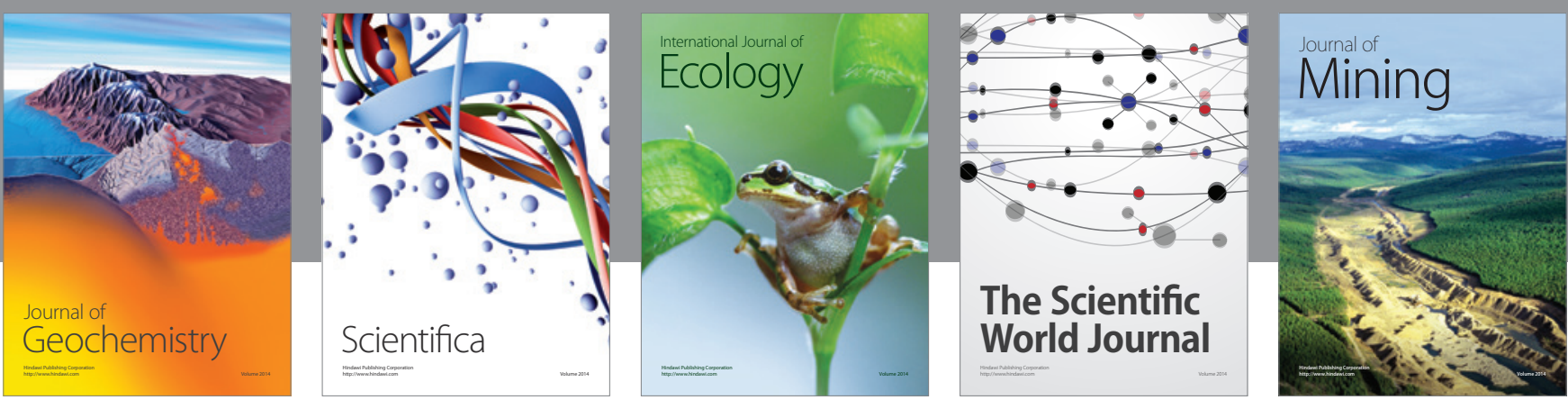

The Scientific World Journal
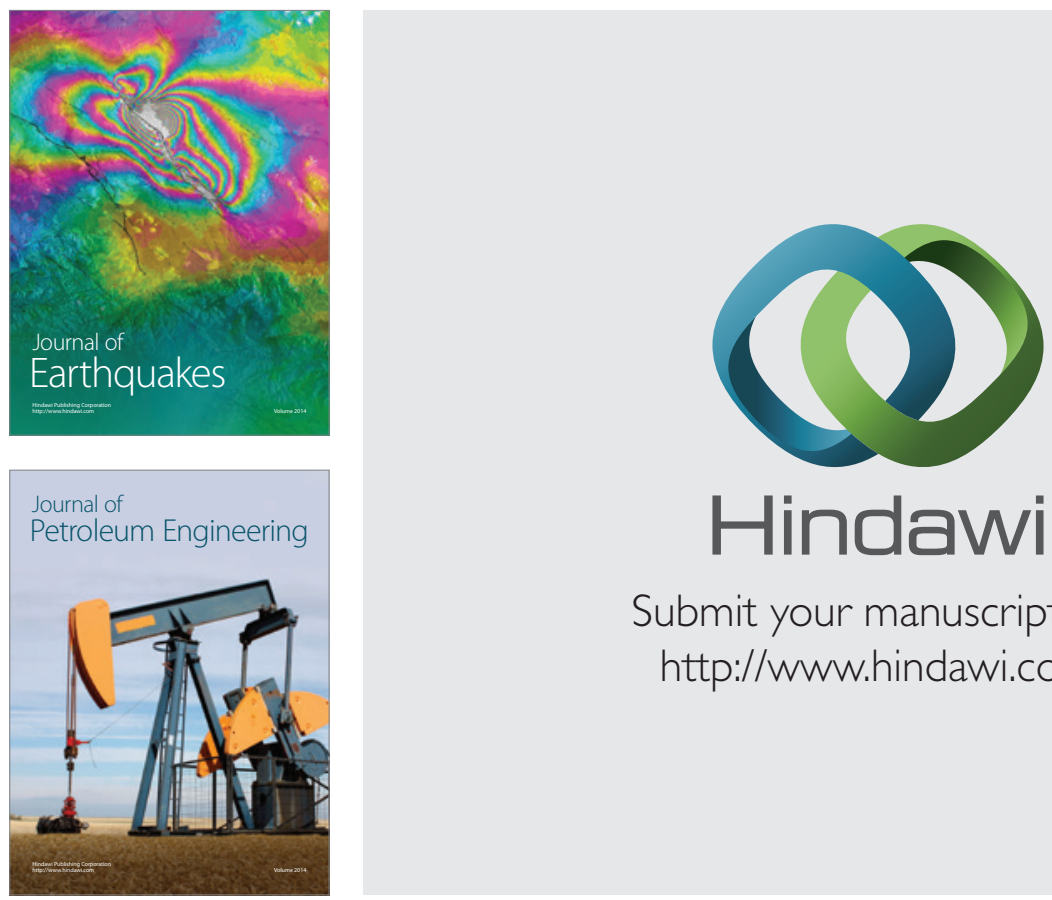

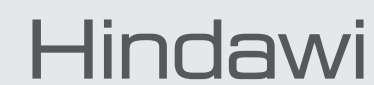

Submit your manuscripts at

http://www.hindawi.com
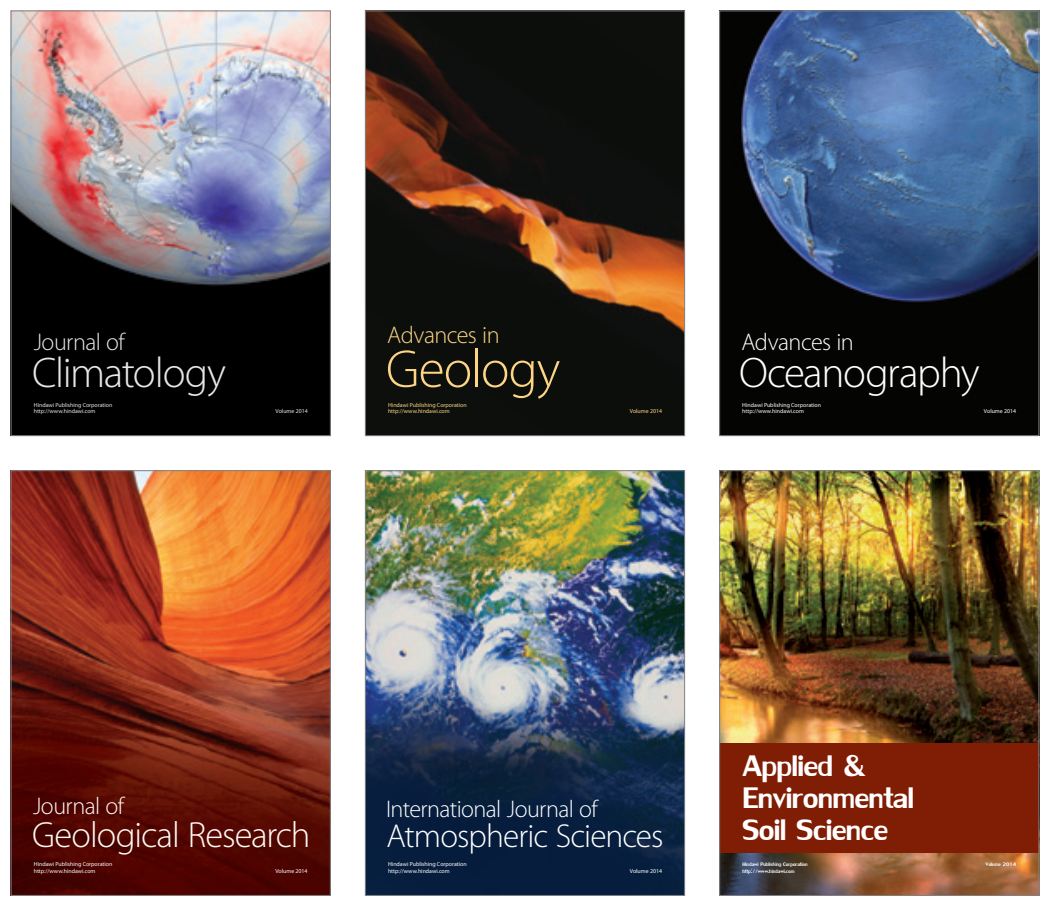
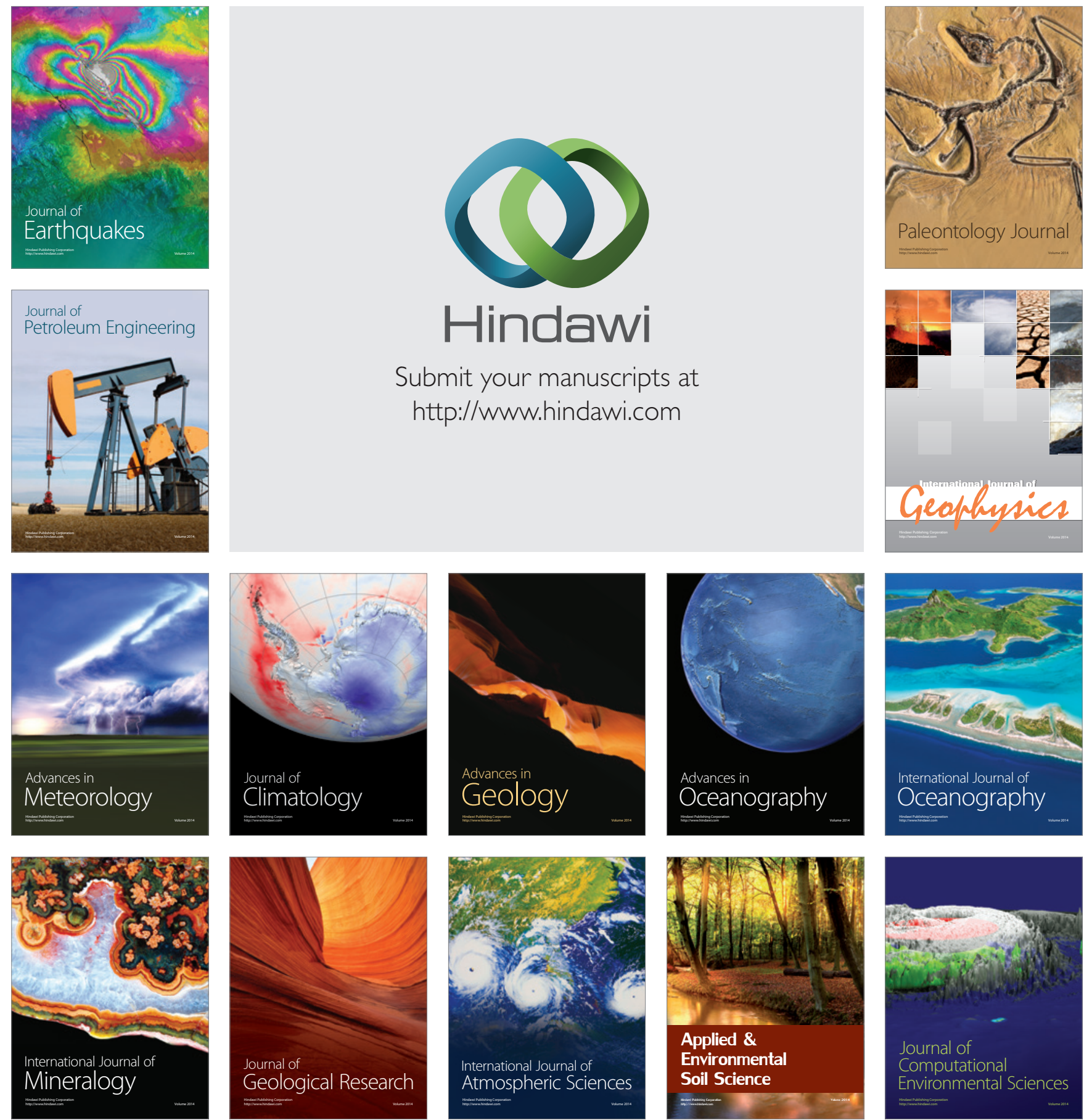